\title{
Formulation of LDL Targeted Nanostructured Lipid Carriers Loaded with Paclitaxel: A Detailed Study of Preparation, Freeze Drying Condition, and In Vitro Cytotoxicity
}

\author{
Jaber Emami, Mahboubeh Rezazadeh, Jaleh Varshosaz, \\ Majid Tabbakhian, and Abolfazl Aslani
}

Department of Pharmaceutics, School of Pharmacy and Pharmaceutical Sciences and Isfahan Pharmaceutical Research Center, Isfahan University of Medical Sciences, Isfahan 73461-8174, Iran

Correspondence should be addressed to Mahboubeh Rezazadeh, rezazade@pharm.mui.ac.ir

Received 11 October 2012; Accepted 13 November 2012

Academic Editor: Li-Hong Liu

Copyright (c) 2012 Jaber Emami et al. This is an open access article distributed under the Creative Commons Attribution License, which permits unrestricted use, distribution, and reproduction in any medium, provided the original work is properly cited.

\begin{abstract}
In the present study, cholesterol nanostructured lipid carriers with various oleic acid content loaded with paclitaxel (PTX) were prepared by solvent emulsification-diffusion method using a Taguchi design. Size, zeta potential, entrapment efficiency, drug loading, and release percent of NLCs were measured. The results indicated that the most effective factors on the size were oleic acid content and surfactant percent. Zeta potential was more affected by the drug content. Drug to- lipid weight ratio was the most effective factor on entrapment efficiency and drug release from NLC. In the present work, the effect of lyophilization on the particle size and release properties of NLCs was also evaluated. The results revealed no differences between the characteristics of NLCs before and after freeze drying by using $25 \% \mathrm{w} / \mathrm{w}$ sorbitol as cryoprotectant. Cytotoxicity studies indicate that PTX associated with the NLC is also effective in HT-29 cell lines and enters the cancer cells selectively through the LDL receptor endocytic pathway. The $\mathrm{IC}_{50}$ values of free PTX solubilized in Cremophor EL and NLC-born PTX after $72 \mathrm{~h}$ exposure were $8.32 \pm 1.35 \mathrm{ng} / \mathrm{mL}$ and $5.24 \pm 0.96 \mathrm{ng} / \mathrm{mL}$, respectively.
\end{abstract}

\section{Introduction}

Paclitaxel (PTX) is one of the most broadly active compounds prescribed to treat aggressive human malignancy such as cancers of ovary, breast, lung, head and neck, esophagus, bladder, endometrium, hematological and pediatric malignancies [1]. Low water solubility of PTX $(0.3 \mu \mathrm{g} / \mathrm{mL})$ and high toxicity have limited its clinical application. Currently, PTX is commercially available as Taxol in a vehicle composed of $1: 1$ of Cremophor EL (CrEL) and ethanol to enhance water solubility of the drug. Some serious side effects associated with CrEL include hypersensitivity reactions, nephrotoxicity, and neurotoxicity. In addition, Taxol has short-term physical stability upon dilution due to the precipitation of PTX in the aqueous media. Other problems with ethanol and CrEL include leaching of plasticizer from polyvinyl chloride infusion bags and sets during injection of the drug [2]. Due to the above-aforementioned limitations, there is a need for new alternative formulations that can overcome poor aqueous solubility of PTX, deliver the drug more specifically to the target organs, and produce fewer side effects. Several approaches such as parenteral emulsions [3], liposomes [4], nanoparticles [57] complex with cyclodextrin [8], water soluble prodrugs [9], and polymeric micelles [10-15] have been employed to deliver PTX into the body or targeted tissue. Solid lipid nanoparticles (SLNs) are one of the carrier systems having more advantages over other colloidal delivery systems. These include biocompatibility, sterility, scale up, and protection of incorporated active ingredients against chemical degradation [16]. However, SLNs are associated with some potential limitations, namely, limited drug loading and drug expulsion during storage due to the crystallization of lipid matrix or lipid polymorphism. To overcome these limitations, nanostructured lipid carriers (NLCs) by mixing solid lipids with chemically very different liquid lipids (oils) have been 
developed to improve drug loading and release properties of conventional SLNs. By using special mixtures of solid lipids and liquid lipids the particles become solid after cooling but do not crystallize which leads to more imperfections in the crystal and higher drug loading [17]. The objective of the present study was to prepare LDL targeted NLCs for delivery of PTX to the cancer cells. In this work for preparation of NLCs, oleic acid (OA) has been used as liquid lipid and cholesterol as solid lipid base. Based on previous published reports, cholesterol-rich nanoemulsion was taken-up by the low-density lipoprotein (LDL) receptors after injection into the bloodstream. Since most cancer cells show LDL receptors upregulation, cholesterol-fabricated nanoparticles can be used as carrier system to target antineoplastic drugs to cancerous cells [18]. Indeed, it was shown in vitro that cholesterol nanoemulsion internalizes drugs such as carmustine, etoposide, and paclitaxel into cultured neoplastic cells [19-21]. Therefore, it is postulated that our NLCs containing cholesterol may concentrate in cancerous cells with LDL receptor overexpression. After injection of the NLCs to the systemic circulation, cholesterol as the lipid base of the nanoparticles acquires apo $\mathrm{E}$ and other apolipoproteins from native lipoproteins. Apo $\mathrm{E}$ that is recognized by LDL receptors allows binding of the artificial NLCs to the receptors and internalize the carrier inside the cancer cells. Nanoemulsions are in liquid form and undergo instabilities such as flocculation, drug release, creaming, gelling, and aggregation of particles; whereas NLCs combine the advantages of polymeric nanoparticles, fat emulsions, and liposome [22]. Lipid nanoparticles that encapsulated paclitaxel have been studied by several groups [23-27]. In most of these studies carriers only achieved passive targeting due to the enhance permeation and retention (EPR) effect. In some of these published reports synergistic combination of passive and active targeting has been provided by chemical functionalizing the surface of nanocarriers with legends such as antibodies, peptides, or small molecules that recognize tumor-specific antigens which is complicated and expensive $[23,24]$. But in our study, using cholesterol simply allows binding of the NLCs to the LDL receptors. This binding mediates internalization of the drug to the neoplastic cells. In this circumstance, the drug is largely released inside the cancer cells resulting enhanced efficiency.

Taguchi design L8 was used to optimize and evaluate the effect of different processing variables on characteristics of the NLCs.

In aqueous dispersed form, NLCs show an increase in particle size after a short period of time. So in subsequent study we aimed to increase the stability of NLCs using freezedrying technique.

The prepared NLCs of PTX could not be lyophilized directly, and use of cryoprotectants was essential to prevent aggregation of the particles after reconstitution. In the current study, the freeze drying of the optimized formulation with or without cryoprotectants was also studied. Furthermore, effects of different variables such as the type and percentage of cryoprotectants and freezing temperature on the characteristics of the particles after freeze drying were also investigated. Moreover, we evaluated the cytotoxic effect
TABle 1: Definition and trial levels of factors in Taguchi L8 orthogonal array experiment used in production of NLCs loaded with PTX.

\begin{tabular}{lcc}
\hline Studied variable & Level 1 & Level 2 \\
\hline Oleic acid content (w/w\%) & 15 & 30 \\
Surfactant type & Poloxamer188 & Tween80 \\
Drug/lipid ratio \% & 5 & 10 \\
Surfactant concentration \% & 1 & 2 \\
Sonication time (min) & 2 & 4 \\
Homogenization rate (rpm) & 800 & 1300 \\
\hline
\end{tabular}

of the drug incorporated in NLCs and compared with those of free drug solutions on the human colorectal cancer cell line, HT-29.

\section{Materials and Methods}

2.1. Materials. Cholesterol, oleic acid, tween80, ethanol, and acetone were obtained from Merck (Germany). Poloxamer 188 and paclitaxel were from Sigma-Aldrich, (US). Avicel RC591 from (FMC, Brussels, Belgium), aerosol from (Evonik, Frankfort Germany), mannitol from (Merk chemical company, Frankfort Germany), dextrose from (Luzhou Bio-Chem, Shandong China), sorbitol, PEG4000 from (Frankfort, Germany), HPLC grade acetonitrile, and methanol from Caledon (Canada). RPMI 1640 medium, sera, and antibiotics for cell culture were purchased from Sigma-Aldrich (US). Human colorectal cancer cells (HT29) were obtained from Pasteur institute (Iran). 3-(4, 5Dimethylthiazol-2-yl)-2, 5-diphenyl tetrazolium bromide (MTT) from Sigma- Aldrich (US).

2.2. Preparation of PTX-Loaded NLC. Different variables along with an overview of the formulations investigated in the present work are listed in Table 2. Different formulations are shown with different codes. The blank-and-drug loaded NLCs were prepared by an emulsion solvent diffusion and evaporation method followed by ultrasonication. Six variables were investigated in two levels. Briefly, $60 \mathrm{mg}$ mixture of cholesterol and oleic acid (OA) at 15 or $30 \mathrm{wt} \%$ OA content and different amount of drug defined according to drug/lipid weight ratio (5 and $10 \mathrm{wt} \%$ PTX) were completely dissolved into the mixture of acetone $(3 \mathrm{~mL})$ and ethanol $(3 \mathrm{~mL})$ in water bath at $60^{\circ} \mathrm{C}$. In the second step, the oily phase was dispersed in an aqueous phase containing a surfactant (poloxamer 188 or tween 80$)$ in different concentrations (1\% or $2 \%$ ) at $60^{\circ} \mathrm{C}$ and premixed by magnetic stirrer for $5 \mathrm{~min}$ at $800 \mathrm{rpm}$. The resulting preemulsion was then ultrasonicated (either at 2 or $4 \mathrm{~min}$ ) using a probe sonicator (Baldelin, Germany) by probe TT13 in amplitude $40 \%$ to produce an $\mathrm{o} / \mathrm{w}$ nanoemulsion. Finally, the obtained nanoemulsion $(\mathrm{o} / \mathrm{w})$ was cooled down at room temperature while stirring on magnetic stirrer at 800 or $1300 \mathrm{rpm}$ for about $1 \mathrm{~h} \mathrm{[27].}$

2.3. Experimental Design and Analysis. Table 1 displays the six independent variables and their levels studied in a L8 orthogonal array using Taguchi design. L and subscript 8 
TABLE 2: Formulations of NLCs produced by a Taguchi design in a standard orthogonal array (L8).

\begin{tabular}{lcccccc}
\hline Code & Wt\% OA & Surfactant type & $\begin{array}{c}\text { Drug/lipid } \\
\text { ratio }\end{array}$ & $\begin{array}{c}\text { Surfactant } \\
\text { conc. }\end{array}$ & $\begin{array}{c}\text { Sonication time } \\
\text { (min) }\end{array}$ & $\begin{array}{c}\text { Homogenization rate } \\
(\mathrm{rpm})\end{array}$ \\
\hline $\mathrm{O}_{15} \mathrm{P}_{1}$ & 15 & Poloxamer188 & 5 & 1 & 2 & 800 \\
$\mathrm{O}_{30} \mathrm{~T}_{2}$ & 30 & Tween80 & 5 & 2 & 2 & 800 \\
$\mathrm{O}_{30} \mathrm{P}_{1}$ & 30 & Poloxamer188 & 10 & 1 & 4 & 800 \\
$\mathrm{O}_{15} \mathrm{P}_{2}$ & 15 & Poloxamer188 & 5 & 2 & 4 & 1300 \\
$\mathrm{O}_{15} \mathrm{~T}_{1}$ & 15 & Tween80 & 10 & 1 & 2 & 1300 \\
$\mathrm{O}_{30} \mathrm{~T}_{1}$ & 30 & Tween80 & 5 & 1 & 4 & 1300 \\
$\mathrm{O}_{30} \mathrm{P}_{2}$ & 30 & Poloxamer188 & 10 & 2 & 2 & 1300 \\
$\mathrm{O}_{15} \mathrm{~T}_{2}$ & 15 & Tween80 & 10 & 2 & 4 & 800 \\
\hline
\end{tabular}

denote the Latin square and the number of the experimental runs, respectively. A run involved the corresponding combination of levels to which the factors in the experiment were set. All experiments were performed in triplicate. Four studied responses were included: particle size, zeta potential, entrapment efficiency (EE), and drug release percent. The experimental results were then analyzed by the Design Expert software version 7 (Stat-Ease, Inc., Minneapolis, Minnesota, USA) to extract independently the main effects of these factors, followed by the analysis of variance (ANOVA) to determine which factors were statistically significant. Identifying controlling factors and qualifying the magnitude of the effects were also emphasized.

\subsection{Determination of Particle Size and Zeta Potential of the} Nanoparticles. Size and zeta potential of NLCs were measured by photon correlation spectroscopy (PCS, Zetasizer 3000, Malvern, UK). All samples were diluted appropriately with the aqueous phase of the formulation for the measurements. $Z$-Average particle size, polydispersity index, and zeta potential were measured in triplicate.

2.5. Analytical Method of PTX Detection. PTX concentration in release medium and its entrapment efficiency in nanoparticles were measured using a reversed-phase HPLC method developed and validated in our laboratory according to a previously published method with a minor modification [27]. The method was validated for linearity, accuracy, and precision. $\mathrm{A} \mathrm{C}_{18}$-Bondapak column $(3.9 \mathrm{~mm} \times 250 \mathrm{~mm})$ was used. The mobile phase consisted of potassium dihydrogen phosphate $(0.01 \mathrm{M})$ /acetonitrile $(45: 55)$ with final $\mathrm{pH}$ adjusted to $3.5 \pm 0.1$ with orthophosphoric acid. The mobile phase eluted at the flow rate of $1.5 \mathrm{~mL} / \mathrm{min}$, and the effluent was monitored at $227 \mathrm{~nm}$ using a UV detector. Column temperature was kept at $40^{\circ} \mathrm{C}$, and $40 \mu \mathrm{L}$ of sample was injected into the HPLC column. Data analysis and processing were performed by millennium software.

2.6. Determination of Entrapment Efficiency and Drug Loading in NLC. Following precipitation of NLCs by centrifugation, the drug content in supernatant was determined in triplicate by HPLC. The separated drug-loaded NLCs were redispersed in $0.1 \mathrm{wt} \%$ tween 80 and surged vertically for 3 min to dissolve the adsorbed drugs on the surface of NLCs.
Then the dispersions were centrifuged at $20000 \mathrm{rpm}$ for another $15 \mathrm{~min}$. The drug content in the supernatant after centrifugation was measured by HPLC method. The drug entrapment efficiency (EE) and drug loading (DL) in the nanoparticles were calculated from (1)

$$
\begin{gathered}
\mathrm{EE}=\left(\frac{W a-W s 1-W s 2}{W a}\right) \times 100, \\
\mathrm{DL}=\left(\frac{W a-W s 1-W s 2}{W a-W s 1-W S 2+W l}\right) \times 100,
\end{gathered}
$$

where $W a$ is the weight of drug added in system, $W s 1$ is the analyzed weight of drug in supernatant after the first centrifugation, $W s 2$ is the analyzed weight of drug in supernatant after the second centrifugation, and $W L$ is the weight of lipid added in system [27].

2.7. In Vitro Release of PTX from NLCs. To determine the release rate of PTX from nanoparticles, $3 \mathrm{~mL}$ of aqueous dispersion of each formulation was added to the dialysis bags with molecular weight cutoff of $12000 \mathrm{Da}$ and the sealed bags were placed in the glass beaker in $150 \mathrm{~mL}$ of the phosphate buffer solution (PBS) $0.1 \mathrm{M}$, PH 7.4 containing $0.1 \%$ tween 80 to provide sink condition with agitation of $200 \mathrm{rpm}$. Samples were withdrawn at predetermined time intervals up to $72 \mathrm{~h}$ and replaced with fresh PBS maintained at the same temperature [28]. The content of PTX in the samples was determined by the described HPLC method.

2.8. Freeze Drying of NLCs. In the preliminary trials the particles were lyophilized by using different types of cryoprotectants: PEG4000, AvicelRC591, aerosil, mannitol, sorbitol, and dextrose. Freezing temperature was $-70^{\circ} \mathrm{C}$ for about 24 hours, and $25 \%(\mathrm{w} / \mathrm{w})$ concentrations of cryoprotectants in mass were used. Operating conditions of the freeze dryer (Christ Alpha 4.2LD over, Germany) were a temperature of $-40^{\circ} \mathrm{C}$ and a pressure of 0.4 bar. After choosing the best cryoprotectant which caused the lowest growth in particle size, subsequently optimization of various effective factors in lyophilization process of NLC of PTX was carried out and the physicochemical properties of the NLCs before and after freeze drying were checked. Redispersion of lyophilized nanoparticles is carried out using $0.01 \%$ PVA in distilled water using a sonicator bath for 10 minutes. 
2.9. Atomic Force Microscopy (AFM). Observation of the morphology and particle size of the optimized NLCs before and after freeze drying was performed by an atomic force microscope (Nanosurf AG, Liestal, Switzerland). AFM images were obtained by measurement of the interaction forces between the tip and the sample surface. The experiments were done in air at room temperature $\left(25^{\circ} \mathrm{C}\right)$ operating in contact mode. Droplets of the final suspension $(20 \mu \mathrm{L})$ were deposited onto a small mica disk. After the drop was dried, the contact mode was used at room temperature.

2.10. Cell Culture. HT-29, the human colorectal adenocarcinoma cell lines, were obtained from Pasteur institute (Iran). Cells were grown at $37^{\circ} \mathrm{C}$ in humidified atmosphere containing 5\% $\mathrm{CO}_{2}$ in RPMI 1640 medium supplemented with $10 \%$ fetal bovine serum (FBS), 100 units/mL penicillin, and 100 units/mL streptomycin. For the experiments, the cells were detached by trypsin treatment, seeded at a density $4 \times 10^{4}$ cells/mL into 96-well culture plate (SPLLifescience, Korea), and incubated for $24 \mathrm{~h}$ at $37^{\circ} \mathrm{C}$ in $5 \% \mathrm{CO}_{2}$ and $100 \%$ humidity before cell viability test.

2.11. In Vitro Cytotoxicity Assay. After seeding the cells on 96 well plate, each row was treated with $20 \mu \mathrm{L}$ of different concentrations of paclitaxel solution (a 50:50 mixture of Cremophor EL and ethanol), $20 \mu \mathrm{L}$ of PTX-loaded NLC formulation in different concentrations, $20 \mu \mathrm{L}$ of blank NLC, $20 \mu \mathrm{L}$ of different concentrations of PTX in DMSO, and $20 \mu \mathrm{L}$ of culture medium (as negative control), respectively. The concentration of paclitaxel varied from 0.1 to $100 \mathrm{ng} / \mathrm{mL}$ for commercial vehicle, NLCs, and DMSO. Then the plate was incubated for $72 \mathrm{~h}$, after that $20 \mu \mathrm{L}$ MTT was added to each well, and cells were incubated for another $4 \mathrm{~h}$. After removing the unreduced MTT and medium, each well was washed with $100 \mu \mathrm{L}$ of PBS, and $180 \mu \mathrm{L}$ of DMSO was then added to each well to dissolve the MTT formazan crystals. Immediately after pipetting, each raw was separately analyzed by ELISA method. Cell viability for each sample was calculated using (2)

Cell survival $=\frac{\text { mean of each group }- \text { mean of blank }}{\text { mean of negative control }- \text { mean of blank }}$.

Another experiment was performed to confirm that cytotoxicity of loaded NLC is mediated by internalization of the drug through LDL receptors. Considering that cholesterol is fitted to the LDL receptors via Apo-E protein, the cellular viability tests were carried out in the absence of Apo-E. In this experiment HT-29 viable cells were grown in RPMI 1640 medium supplemented with 100 units/mL penicillin, 100 units/mL streptomycin and without serum. After that, the cells were detached by trypsin treatment, seeded at a density $4 \times 10^{4}$ cells $/ \mathrm{mL}$ into 96 -well culture plate, and incubated for $24 \mathrm{~h}$ at $37^{\circ} \mathrm{C}$ in $5 \% \mathrm{CO}_{2}$ and $100 \%$ humidity. After this period, $10 \mathrm{ng} / \mathrm{mL}$ PTX, free or associated with NLC, was added to the plate in triplicate and incubated for $24 \mathrm{~h}$ at $37^{\circ} \mathrm{C}$. The cell cytotoxicity and survival percent were evaluated by MTT assay.

\section{Results and Discussion}

3.1. Particle Size and Zeta Potential of NLCs. Solvent diffusion method was used for preparation of PTX-loaded NLC. The organic phase was added to the aqueous phase in the same temperature at $60^{\circ} \mathrm{C}$. $\mathrm{Hu}$ et al. have shown that the lower temperature of dispersion medium led to the lower diffusion rate of organic dispersion phase and consequently formed the relatively large particles with wide size distribution [29]. The properties of PTX-loaded NLCs such as particle size, polydispersity index (PI), zeta potential, entrapment efficiency, and release percent are listed in Table 3 .

It was found that the size of drug-loaded particles was higher than that of drug-free ones due to the incorporation of drug in NLC matrix. On the other hand, the most effective factor on particle size relates to the drug content. Changing the drug content from 5 to 10 increased the size of nanoparticles effectively.

As shown in Figure 1, the OA content and surfactant concentration had also rather high contribution to the particle size. The higher oleic acid content reduced the viscosity inside NLC, and consequently, reduced the surface tension to form smaller particles. The polydispersity index was also decreased by increasing the OA content. These results suggested that the addition of $\mathrm{OA}$ to the formulations was in favor of forming nanoparticles with more homogenous size distributions due to the size reduction. In the study conducted by $\mathrm{Hu}$ et al., stearic acid nanostructured lipid carriers with various oleic acid content loaded by clobetasol were prepared by solvent diffusion method. In their study as OA content was increased up to $30 \%$, the particle sizes were decreased with more regular morphology which is in agreement with our results [30]. Increasing the concentration of surfactant decreased the particle sizes of nanoparticles. Muller has shown that increasing the poloxamer concentration to $1 \%$ was effective in producing smaller size SLN composed of tripalmitin, cetylpalmitate, and glycerylmonostearate [31]. In present study, it seems that $1 \%$ poloxamer is sufficient to cover the surface of nanoparticles effectively and prevents agglomeration during the process.

Zeta potential is often a key factor to understand the stability of colloidal dispersion. As shown in Table 3 the absolute value of zeta potential in most formulation was above 10. This demonstrates that the nanoparticles dispersion obtained by solvent diffusion method in an aqueous system in this study is a physically stable system [32]. The data shown in Figure 1 reveals that the drug content is the most effective variable on zeta potential of nanoparticles. Changing drug/lipid weight ratio from level 1 to level 2 (increasing the drug percent from 5\% to 10\%) decreased the absolute value of zeta potential. Freitas and Muller have shown that the increased drug content could reduce the charge density and absolute values of zeta potential [33]. Our results revealed that changing the surfactant type from tween 80 to poloxamer 188 elevated the absolute value of the zeta potential. In the study conducted by Lim and Kim the zeta potential was decreased in the corporation of tween 80 
TABLE 3: Physical properties of different NLCs.

\begin{tabular}{lcccccc}
\hline Code & $\begin{array}{c}\text { Particle size } \\
(\mathrm{nm})\end{array}$ & Pd index & $\begin{array}{c}\text { Zeta potential } \\
(\mathrm{mV})\end{array}$ & EE* \% & $\begin{array}{c}\text { DL** \% } \\
\text { \% }\end{array}$ & $\begin{array}{c}\text { Drug released } \\
\text { after } 72 \mathrm{~h}\end{array}$ \\
\hline $\mathrm{O}_{15} \mathrm{P}_{1}$ & $158.1 \pm 17.1$ & $0.1 \pm 0.009$ & $-20.8 \pm 0.26$ & $63 \pm 13.2$ & $3.3 \pm 0.5$ & $43.39 \pm 6.8$ \\
$\mathrm{O}_{30} \mathrm{~T}_{2}$ & $80.68 \pm 15.9$ & $0.2 \pm 0.01$ & $-9.1 \pm 0.12$ & $72 \pm 11.6$ & $2.8 \pm 0.3$ & $53.9 \pm 7.6$ \\
$\mathrm{O}_{30} \mathrm{P}_{1}$ & $173.6 \pm 19.8$ & $0.3 \pm 0.02$ & $-12.7 \pm 0.19$ & $60 \pm 10.3$ & $2.8 \pm 0.5$ & $59.3 \pm 5.6$ \\
$\mathrm{O}_{15} \mathrm{P}_{2}$ & $132.9 \pm 21.3$ & $0.2 \pm 0.04$ & $-19.7 \pm 0.23$ & $63 \pm 9.8$ & $2.3 \pm 0.4$ & $52.23 \pm 4.3$ \\
$\mathrm{O}_{15} \mathrm{~T}_{1}$ & $189.4 \pm 15.6$ & $0.6 \pm 0.02$ & $-9.17 \pm 0.15$ & $46 \pm 10.2$ & $2.1 \pm 0.2$ & $50.5 \pm 8.5$ \\
$\mathrm{O}_{30} \mathrm{~T}_{1}$ & $100.9 \pm 12.3$ & $0.3 \pm 0.01$ & $-12.9 \pm 0.15$ & $62 \pm 11.6$ & $3.5 \pm 0.2$ & $50.23 \pm 6.3$ \\
$\mathrm{O}_{30} \mathrm{P}_{2}$ & $137.3 \pm 13.4$ & $0.1 \pm 0.03$ & $-8.8 \pm 0.13$ & $53 \pm 8.6$ & $2.9 \pm 0.5$ & $70.3 \pm 5.4$ \\
$\mathrm{O}_{15} \mathrm{~T}_{2}$ & $169.8 \pm 10.8$ & $0.4 \pm 0.04$ & $-10.7 \pm 0.09$ & $53 \pm 7.3$ & $3.1 \pm 0.6$ & $63.78 \pm 8.1$ \\
\hline
\end{tabular}

${ }^{*}$ EE: entrapment efficiency. ${ }^{* *}$ DL: drug loading.

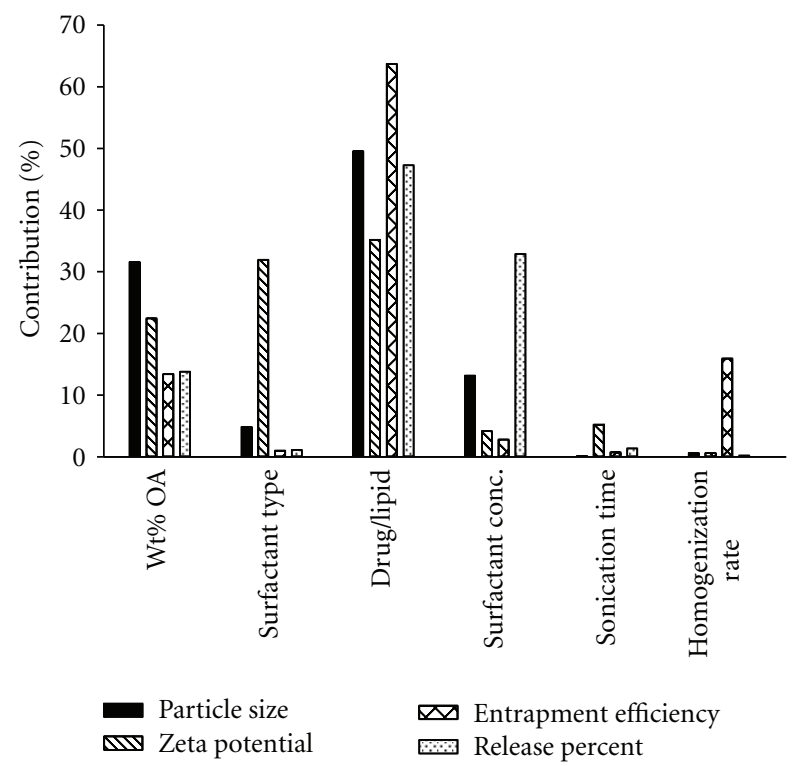

Figure 1: Contribution percent of different effective factors on the particle size, zeta potential, entrapment efficiency, and release percent of NLCs loaded with PTX.

as surfactant [34]. The results of Table 3 and data analysis showed that absolute zeta potential decreased as emulsifier concentration increased to $2 \%$ which was in accordance with the results of other researchers [35]. Data analysis showed that increasing the amount of OA could decrease the absolute value of zeta potential. In preparation of clobetasol NLCs with various amount of oleic acid, no trend was found for zeta potential changes by increasing the OA content of nanoparticles [30]. In fact crystalline reorientation or recrystallization of lipid can result in changes on the particle surface and subsequently change the zeta potential unpredictably.

3.2. Entrapment Efficiency in NLC. The calibration curve of HPLC method used for determination of drug content and drug release studies was linear in the concentration range $0.05-2 \mu \mathrm{g} / \mathrm{mL}\left(r^{2}=0.999\right)$. The inter- and intraday precision and accuracy were less than $10.27 \%$ with the detection limit $20 \mathrm{ng} / \mathrm{mL}$. As Figure 1 shows, drug to lipid weight ratio is the most effective factor on entrapment efficiency in NLC. Changing the drug/lipid weight ratio from $10 \%$ to $5 \%$, decreasing the amount of drug to lipid, accompanied good entrapment of drug in the lipids. The OA content were also influenced loading and entrapment efficiency. The drug entrapment efficiency was increased, by increasing the percentage of OA. Previous studies have illustrated that incorporation of liquid lipids to solid lipids can lead to massive crystal order disturbances. This results in great imperfections in the crystal lattice, which in turn leaves enough space to accommodate drug molecules, which ultimately improves drug loading capacity and drug entrapment efficiency [36]. As shown in Figure 1, other factors were not effective on the drug loading.

3.3. In Vitro Release of PTX from NLC. Figure 1 shows that the drug release from NLCs is more affected by drug/lipid ratio and the surfactant concentration. The release rate became faster when the amount of $\mathrm{OA}$ was increased. Surfactant type, sonication time, and homogenization rate did not significantly affect the release rate. The drug release profiles from NLCs are shown in Figure 2. Drug-to-lipid weight ratio could influence the release of PTX from nanoparticles. When the drug increased from 5 to $10 \%$, most of the drug located at the outer shell of nanoparticles which led to faster drug release. For all formulations, a biphasic drug release pattern was observed, which is due to the burst release at the initial stage and is followed by sustained release in the later times. The release rate in initial stage became faster with increasing the OA content. On the other hand, when the OA content increased from 15 to $30 \%$, the particle size decreased; consequently, the specific surface area was increased, and the faster release rate was observed. Changing the surfactant concentration from level1 to 2 also increased the drug release rate.

3.4. Optimization of PTX-NLCs. Computer optimization process and a desirability function determined the effect of the levels of independent variables on the responses. All responses were fitted to the linear model. The constraints of particle size were 80.68 to $189.4 \mathrm{~nm}$ with targeting the particle size in the range of $100-189$, for zeta potential was 


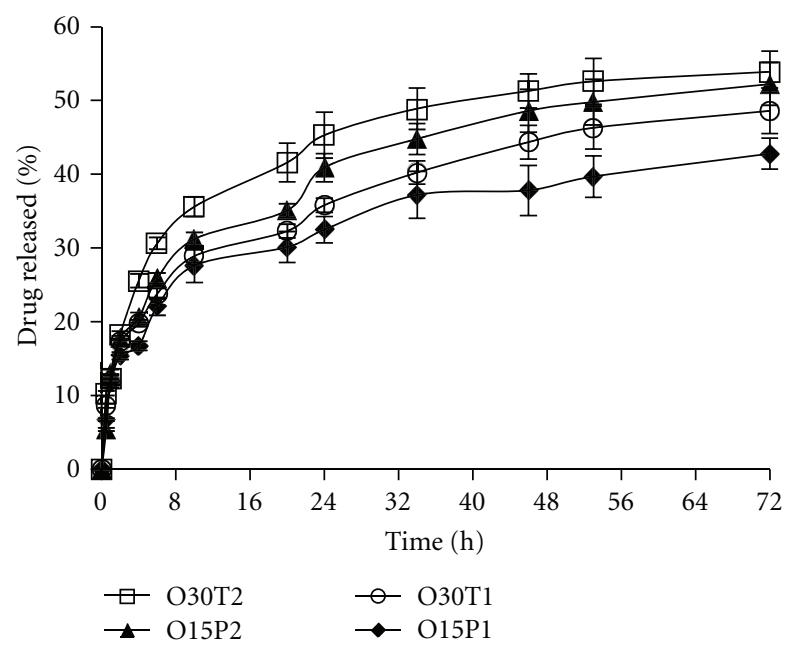

(a)

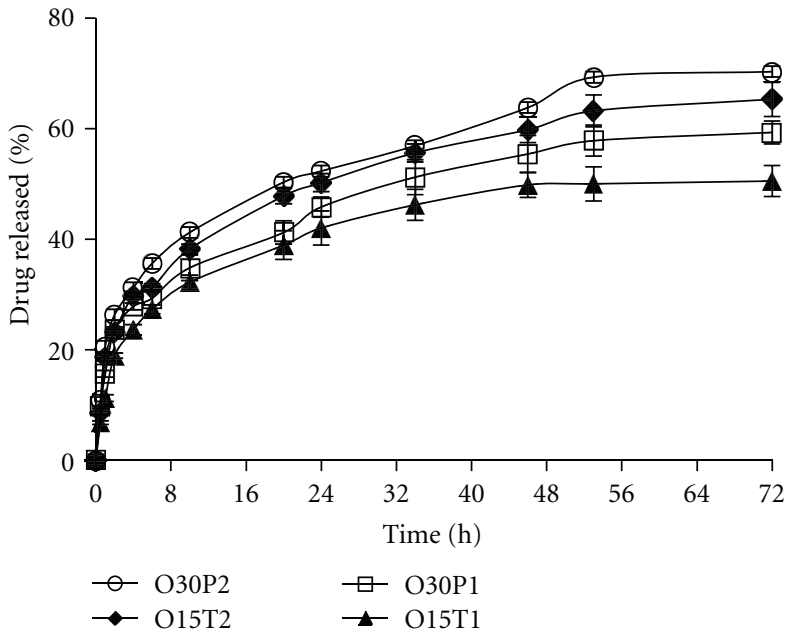

(b)

Figure 2: In vitro release profile of paclitaxel from cholesterol NLCs $(n=3)$ : (a) drug/lipid 5\% w/w, (b) drug/lipid 10\% w/w.

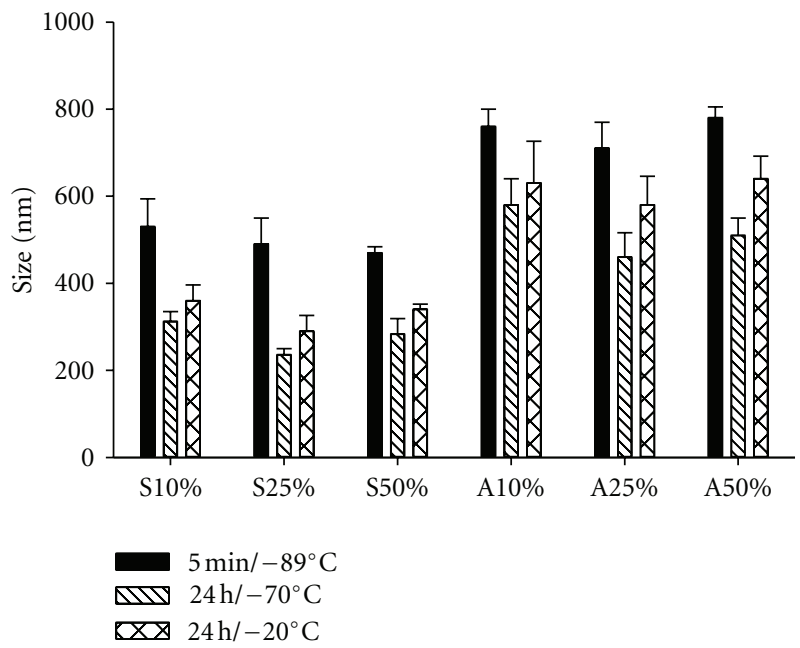

Figure 3: Effect of different cryoprotectants, concentration, and freezing rate on particle size. Sublimation time did not have significant effect on particle size. S: Sorbitol, A: Aerosil.

-8.8 to $-20.8 \mathrm{mV}$, while the target was maximum absolute value of zeta potential, for the EE the constraint was 46 to $72 \%$ with the goal of maximum value, and for release percent the target was considered in the range of the measured value.

Accordingly, the predicted optimized formulation by the software would contain 5\% PTX, 30\% OA, 1\% poloxamer188, 2 minute sonication, and $800 \mathrm{rpm}$ homogenization rate. To confirm the predicted model, the optimized formulation was prepared, and the observed responses were measured and listed in Table 4 . The acceptable agreement between the observed values and the values predicted by the software and the negligible error percent confirm the validation and reliability of our method as well as its adequate
TABle 4: Predicted versus actual responses obtained for the optimized formulation.

\begin{tabular}{lcccc}
\hline Responses & $\begin{array}{c}\text { Particle size } \\
(\mathrm{nm})\end{array}$ & $\begin{array}{c}\text { Zeta potential } \\
(\mathrm{mV})\end{array}$ & $\begin{array}{c}\text { Release after } \\
72 \mathrm{~h}\end{array}$ & $\mathrm{EE}(\mathrm{h})$ \\
\hline $\begin{array}{l}\text { Actual } \\
\text { values }\end{array}$ & $181.6 \pm 14.8$ & $-12.9 \pm 0.2$ & $42.3 \pm 3.6$ & $53 \pm 4.2$ \\
$\begin{array}{l}\text { Predicted } \\
\text { values }\end{array}$ & 142.33 & -12.33 & 50 & 59 \\
Error $(\%)$ & 21.54 & 4.41 & 18.77 & 11.32 \\
\hline
\end{tabular}

TABLE 5: Results of a preliminary screening of different cryoprotectants.

\begin{tabular}{lcc}
\hline Cryoprotectant & $\begin{array}{c}\text { Particle size } \\
(\mathrm{nm})\end{array}$ & $\begin{array}{c}\text { Zeta potential } \\
(\mathrm{mV})\end{array}$ \\
\hline PEG4000 & $1550 \pm 296.3$ & $-4.5 \pm 0.3$ \\
Avicel RC591 & $956 \pm 120.6$ & $-6.9 \pm 0.9$ \\
Mannitol & $1056 \pm 213.6$ & $-2.8 \pm 0.1$ \\
Dextrose & $857 \pm 87.6$ & $-4.3 \pm 0.4$ \\
Sorbitol & $216 \pm 21.2$ & $-10.6 \pm 0.7$ \\
Aerosil & $480 \pm 83.2$ & $-15.6 \pm 0.3$ \\
\hline
\end{tabular}

precision for the prediction of optimized conditions in the domain of levels chosen for the independent variables.

3.5. Freeze Drying of NLCs. As mentioned, lyophilization is an approach to improve the physical and chemical stability of colloidal system. The results of the preliminary screening study of different cryoprotectants listed in Table 5 show that PEG4000, Avicel, and dextrose cause an increase in size to about 1 microns and zeta potential to about $-5 \mu \mathrm{m}$ suggesting an aggregation of particles, leading to poor redispersibility. In a work conducted by varshosaz et al., PEG as cryoprotectant caused a significant increase in size and zeta potential [32]. 


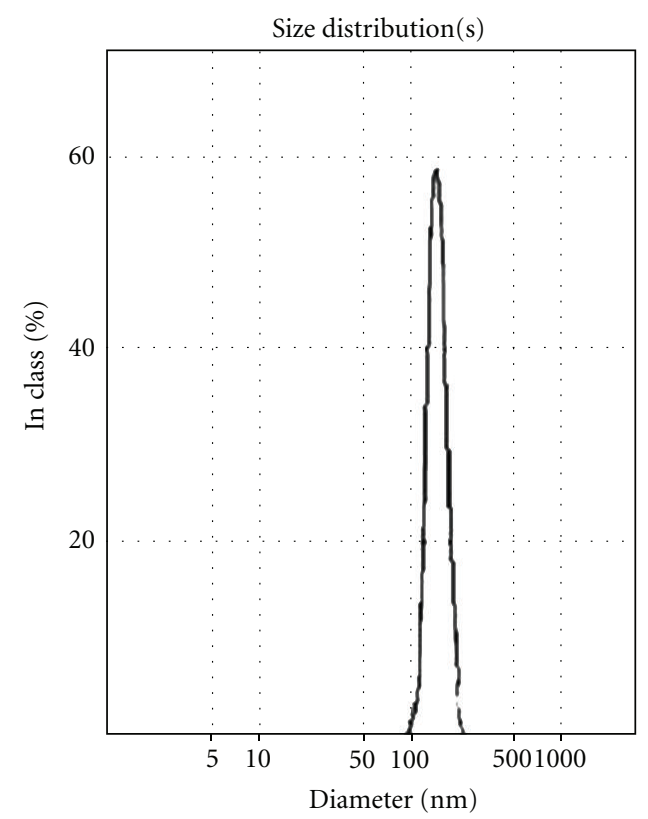

(a)

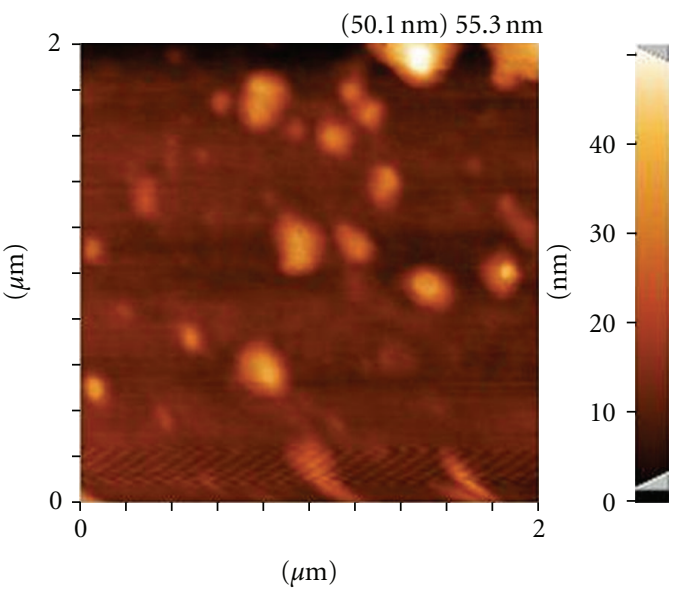

(c)

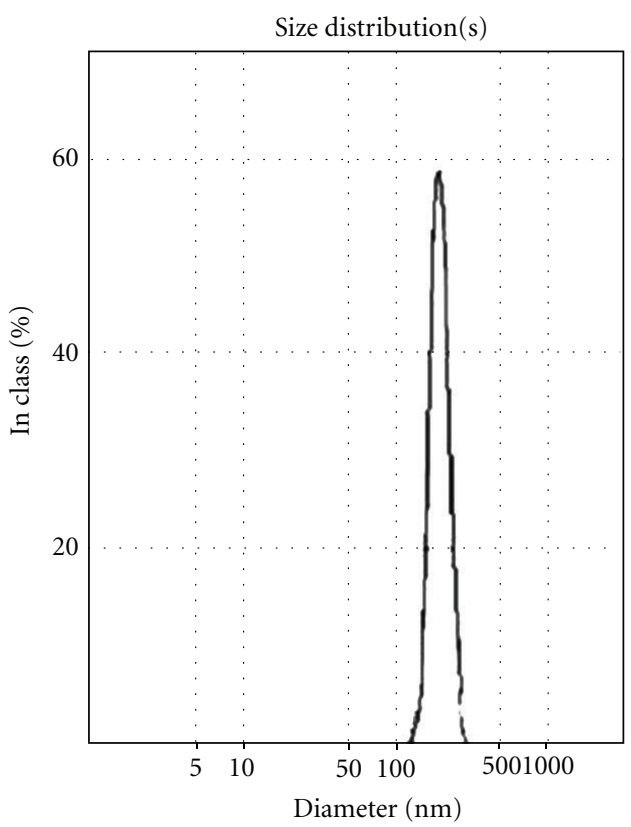

(b)

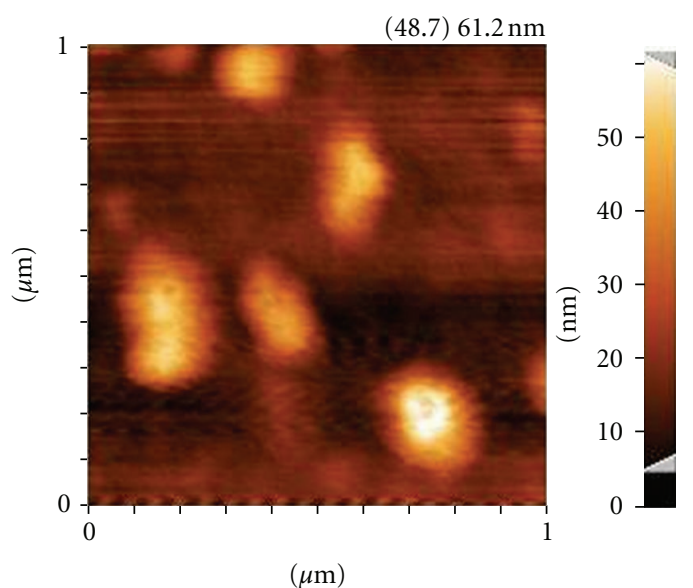

(d)

FIGURE 4: size distribution and AFM images of NLCs of PTX (a) size distribution of NLCs before freeze drying, (b) size distribution of NLCs after freeze drying, (c) AFM image of NLCs before freeze drying, and (d) AFM image of NLCs after freeze drying.

Mannitol also showed poor redispersibility with particle size greater than 1 micron which may be related to crystallization of mannitol in low temperature. Moreover, the growing crystals of water and mannitol may exert mechanical forces on the nanoparticles leading to their fusion. Table 5 shows that sorbitol and aerosil cause less increase in particle size and zeta potential after redispersion of dried nanoparticles in distilled water. However, to optimize the lyophilization process the effect of concentration and freezing rate should be considered. In the literature, different freezing methods have been used to freeze nanoparticle suspensions. In this study we used liquid nitrogen freezing $\left(-89^{\circ} \mathrm{C} / 5 \mathrm{~min}\right)$ and loading vials onto precooled shelves $\left(-70^{\circ} \mathrm{C} / 24 \mathrm{~h},-20^{\circ} \mathrm{C} / 24 \mathrm{~h}\right)$.

The results showed an increase in the particle size of NLCs using rapid freezing rate for all the tested formulations.
However, some studies have shown that high super cooling leads to the formation of small ice crystals and may decrease the mechanical stress on nanoparticles avoiding their aggregations. Our results may be explained by the fact that quick freezing in liquid nitrogen destabilized the nanoparticles leading to a greater formation of aggregates after redispersion, so slower freezing at $-70^{\circ} \mathrm{C}$ in deep freezer proved to be better method for this formulation. In the study conducted by Saez et al., sorbitol was found to be an effective cryoprotectant when PLGA nanoparticles were frozen at $-70^{\circ} \mathrm{C}$ [37]. We also investigated the effect of cryoprotectant concentrations in three levels $(10,25$, and $50 \% \mathrm{w} / \mathrm{w})$. As shown in Figure 3, it seems that sorbitol was better than aerosil in reducing particle size. Sorbitol in $25 \% \mathrm{w} / \mathrm{w}$ concentration may arise from the formation 


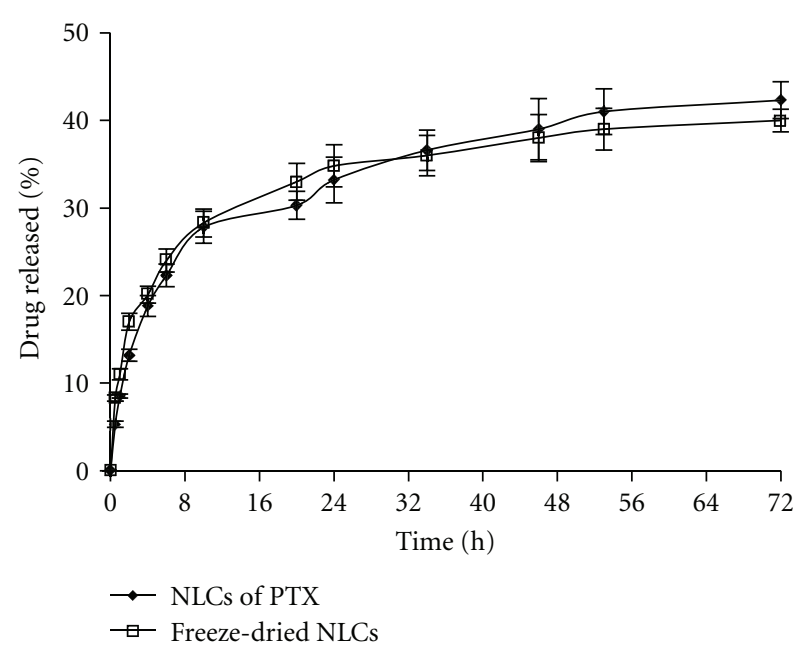

FIGURE 5: Release profiles of PTX from NLCs before and after freeze drying.

of a protective capping layer around the NLCs. The zeta potential range for all preparation was low, with the range -9.8 up to $-15.5 \mathrm{mv}$. This may be related to the effect of PVA. PVA is a polymer with long-chain alkyl which causes a negative charge. Redispersion of lyophilized formulations in $0.01 \%$ PVA reduces the zeta potential and improves dispersion. Our results also revealed that aerosil causes more decrease in zeta potential than sorbitol. Perhaps because the suspension of aerosil has negative zeta potential and increases the absolute zeta potential of nanoparticles, while sorbitol is a natural compound that dissolves in water.

Based on our results, the optimum condition is obtained using sorbitol $25 \% \mathrm{w} / \mathrm{w}$ concentration, freezing rate of $-70^{\circ} \mathrm{C} / 24 \mathrm{~h}$, and $48 \mathrm{~h}$ sublimation time. To confirm the data of particle size, the AFM photographs and size distributions of the nanoparticles prior and after freeze drying by using $25 \%$ sorbitol were also shown in Figure 4. Optimized freeze drying condition maintained the particle size of NLCs of PTX after redispersion. The AFM images of the nanoparticles revealed spherical and round shape of approximately 150$200 \mathrm{~nm}$ in diameter, which was similar to the results obtained by photon correlation spectroscopy. The results of loading percent and 3 days drug release study on optimized formulation after redispersion showed $42 \%$ drug loading and $40 \%$ drug release from nanoparticles. As shown in Figure 5 there is no significant change in the burst effect and drug release content from NLCs before and after lyophilization.

3.6. In Vitro Cytotoxicity. Figure 6 shows the cytotoxic activity curves of the optimized NLC-PTX formulation, PTX solubilized in DMSO, PTX in Creomophor EL, and blank NLC. The blank NLCs did not show significant cytotoxicity whit more than $80 \%$ of HT-29 cells survived after 72 exposure to the highest concentration of blank NLCs.

At low concentration $(0.1 \mathrm{ng} / \mathrm{mL})$, only free PTX in DMSO and Cr EL was effective to suppress the cell growth, while PTX loaded-NLCs displayed no cytotoxicity, which may be attributed to the relatively low amount of drug

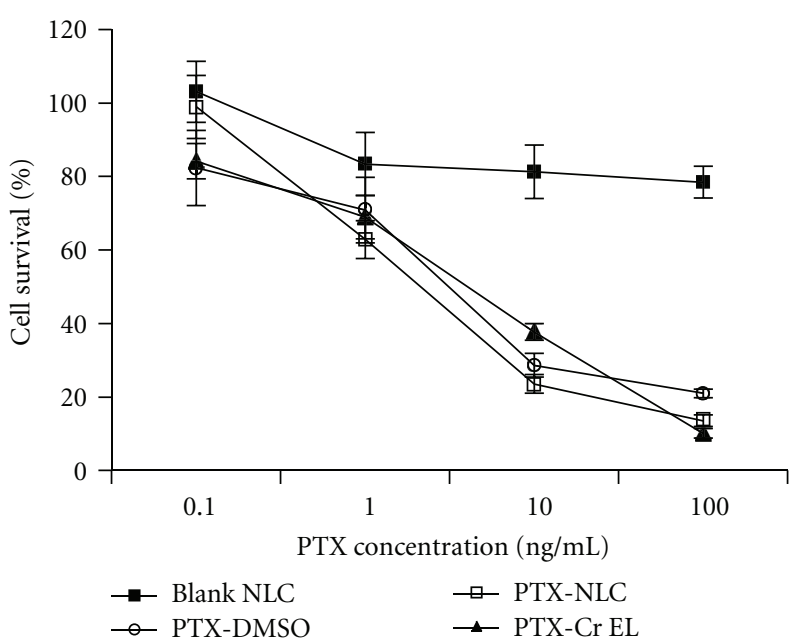

FIGURE 6: In vitro cytotoxicity of various PTX formulations and blank NLC against HT-29 cell line after $72 \mathrm{~h}$ incubation. Data were plotted as the mean \pm SD of three measurements.

TABLE 6: IC $_{50}$ values of PTX solutions and the drug-loaded NLC against HT-29 cell lines.

\begin{tabular}{lccc}
\hline \multirow{2}{*}{ Drug formulation } & \multicolumn{3}{c}{$\mathrm{IC}_{50}($ mean $\pm \mathrm{SD})$} \\
& $24 \mathrm{~h}$ & $48 \mathrm{~h}$ & $72 \mathrm{~h}$ \\
\hline PTX in cremophor EL & $15.81 \pm 3.12$ & $10.23 \pm 1.56$ & $8.32 \pm 1.35$ \\
PTX-NLC & $18.93 \pm 2.56$ & $11.24 \pm 2.34$ & $5.24 \pm 0.96$ \\
PTX in DMSO & $13.31 \pm 4.12$ & $8.93 \pm 3.56$ & $6.91 \pm 0.85$ \\
\hline
\end{tabular}

released from the carriers. At high concentrations (1100 ng/mL) PTX-loaded NLCs also showed decrease in cell growth, which was comparable to free PTX. Thus our finding demonstrated that PTX-NLC developed in this study had the potency similar to that of Cremophor-EL-based formulation and could be a safe drug carrier. The amount of PTX required to achieve the $\mathrm{IC}_{50}$ values were evaluated after 24,48 , and $72 \mathrm{~h}$ exposure of cells to the free PTX and PTX-loaded NLCs. As shown in Table 6 the cytotoxicity of PTX was time dependent.

Survival of HT-29 cultured in the absence of Apo E was considerably increased by incubation with NLC-PTX. On the other hand, free drug cytotoxicity was much less affected by the change in growth medium (Figure 7). It is concluded that the drug associated with cholesterol lipid base is uptook into the cancer cells by endocytosis via LDL receptors, and when Apo $\mathrm{E}$ is removed from the serum in the medium of cell culture the targeting to the LDL receptors is blocked and cell viability is increased. The unassociated drug could freely cross the cellular membrane and exert its cytotoxicity. As expected the cytotoxicity of free drug was unaffected by the absence of Apo E from culture medium. Hence, the optimized NLCs developed in our study could be an efficient vehicle for delivery of PTX to the intended site without provoking any side effect associated with cremophor EL containing formulations. The drug content from these lipidic nanoparticles is largely released inside the cancer 


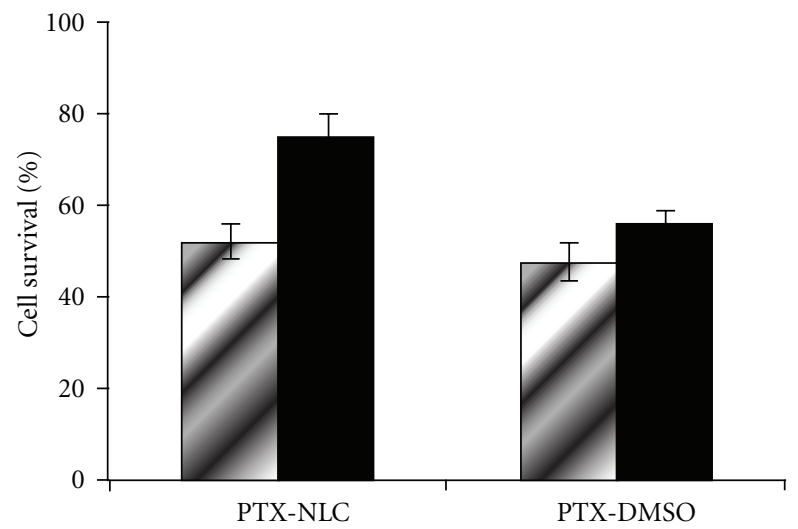

Figure 7: Cytotoxicity of PTX-loaded NLC and free PTX in the presence (grey columns) or absence (black columns) of Apo E after $24 \mathrm{~h}$ incubation.

cells resulting in an enhanced efficiency and reducing side effects.

\section{Conclusion}

In the present study the PTX-loaded NLCs were prepared using solvent diffusion method following probe sonication and finally freeze dried under certain conditions which were evaluated by different factors. Optimized formulation containing 5\% PTX, 30\% OA, $1 \%$ poloxamer 188,2 minute sonication, and $800 \mathrm{rpm}$ homogenization rate gave nanoparticles with about $181.6 \mathrm{~nm}$ with 0.1 polydispersity, $-12.9 \mathrm{Mv}$ zeta potential, $43 \%$ drug release after 3 days and $53 \%$ entrapment efficiency. The optimum condition in freeze drying was achieved by using sorbitol in $25 \%$ $\mathrm{w} / \mathrm{w}$, freezing rate of $-70 / 24$, and sublimation time $48 \mathrm{~h}$. Freeze-dried NLCs suspended in PVA $0.01 \%$ solution still were spherical with the mean diameter about $200 \mathrm{~nm}$ and entrapment efficiency $42 \%$. cytotoxicity results showed that PTX associated with NLCs is also effective and exerts the pharmacologic effect by entering the cell through the LDL receptor endocytic pathway. Thus, the optimized NLCs developed in our study could deliver PTX selectively to the cancer cells without any side effect associated with cremophor EL containing formulations. These nanoparticles are, therefore, anticipated to be a promising PTX carrier for their targeted and intracellular delivery.

\section{References}

[1] A. W. G. Alani, Y. Bae, D. A. Rao, and G. S. Kwon, "Polymeric micelles for the $\mathrm{pH}$-dependent controlled, continuous low dose release of paclitaxel," Biomaterials, vol. 31, no. 7, pp. 1765-1772, 2010.

[2] J. H. Kim, Y. S. Kim, S. Kim et al., "Hydrophobically modified glycol chitosan nanoparticles as carriers for paclitaxel," Journal of Controlled Release, vol. 111, no. 1-2, pp. 228-234, 2006.

[3] A. O. Nornoo, D. W. Osborne, and D. S. L. Chow, "Cremophor-free intravenous microemulsions for paclitaxel. I: formulation, cytotoxicity and hemolysis," International Journal of Pharmaceutics, vol. 349, no. 1-2, pp. 108-116, 2008.
[4] G. J. Fetterly and R. M. Straubinger, "Pharmacokinetics of paclitaxel-containing liposomes in rats," AAPS PharmSci, vol. 5, no. 4, pp. 90-100, 2003.

[5] M. J. Hawkins, P. Soon-Shiong, and N. Desai, "Protein nanoparticles as drug carriers in clinical medicine," Advanced Drug Delivery Reviews, vol. 60, no. 8, pp. 876-885, 2008.

[6] F. Danhier, N. Lecouturier, B. Vroman et al., "Paclitaxel-loaded PEGylated PLGA-based nanoparticles: in vitro and in vivo evaluation," Journal of Controlled Release, vol. 133, no. 1, pp. 11-17, 2009.

[7] F. Lacoeuille, F. Hindre, F. Moal et al., "In vivo evaluation of lipid nanocapsules as a promising colloidal carrier for paclitaxel," International Journal of Pharmaceutics, vol. 344, no. 1-2, pp. 143-149, 2007.

[8] U. S. Sharma, S. V. Balasubbamanian, and R. M. Straubinger, "Pharmaceutical and physical properties of paclitaxel (Taxol) complexes with cyclodextrins," Journal of Pharmaceutical Sciences, vol. 84, no. 10, pp. 1223-1230, 1995.

[9] B. Elsadek, R. Graeser, N. Esser et al., "Development of a novel prodrug of paclitaxel that is cleaved by prostate-specific antigen: an in vitro and in vivo evaluation study," European Journal of Cancer, vol. 46, no. 18, pp. 3434-3444, 2010.

[10] X. Zhang, J. K. Jackson, and H. M. Burt, "Development of amphiphilic diblock copolymers as micellar carriers of taxol," International Journal of Pharmaceutics, vol. 132, no. 1-2, pp. 195-206, 1996.

[11] S. C. Kim, D. W. Kim, Y. H. Shim et al., "In vivo evaluation of polymeric micellar paclitaxel formulation: toxicity and efficacy," Journal of Controlled Release, vol. 72, no. 1-3, pp. 191-202, 2001.

[12] T. Hamaguchi, Y. Matsumura, M. Suzuki et al., "NK105, a paclitaxel-incorporating micellar nanoparticle formulation, can extend in vivo antitumour activity and reduce the neurotoxicity of paclitaxel," British Journal of Cancer, vol. 92, no. 7, pp. 1240-1246, 2005.

[13] C. Zhang, P. Qineng, and H. Zhang, "Self-assembly and characterization of paclitaxel-loaded N-octyl-O-sulfate chitosan micellar system," Colloids and Surfaces B, vol. 39, no. 1-2, pp. 69-75, 2004.

[14] F. Q. Hu, G. F. Ren, H. Yuan, Y. Z. Du, and S. Zeng, "Shell cross-linked stearic acid grafted chitosan oligosaccharide self-aggregated micelles for controlled release of paclitaxel," Colloids and Surfaces B, vol. 50, no. 2, pp. 97-103, 2006.

[15] M. Huo, Y. Zhang, J. Zhou, A. Zou, and J. Li, "Formation, microstructure, biodistribution and absence of toxicity of polymeric micelles formed by N-octyl-N,O-carboxymethyl chitosan," Carbohydrate Polymers, vol. 83, no. 4, pp. 19591969, 2011.

[16] R. H. Müller, K. Mäder, and S. Gohla, "Solid lipid nanoparticles (SLN) for controlled drug delivery-a review of the state of the art," European Journal of Pharmaceutics and Biopharmaceutics, vol. 50, no. 1, pp. 161-177, 2000.

[17] R. H. Müller, M. Radtke, and S. A. Wissing, "Nanostructured lipid matrices for improved microencapsulation of drugs," International Journal of Pharmaceutics, vol. 242, no. 1-2, pp. 121-128, 2002.

[18] K. V. Pinheiro, V. T. M. Hungria, E. S. Ficker, C. J. Valduga, C. H. Mesquita, and R. C. Maranhão, "Plasma kinetics of a cholesterol-rich microemulsion (LDE) in patients with Hodgkin's and non-Hodgkin's lymphoma and a preliminary study on the toxicity of etoposide associated with LDE," Cancer Chemotherapy and Pharmacology, vol. 57, no. 5, pp. 624-630, 2006. 
[19] R. C. Maranh, S. R. Graziani, and N. Yamaguchi, "Association of carmustine with a lipid emulsion: in vitro, in vivo and preliminary studies in cancer patients," Cancer Chemotherapy and Pharmacology, vol. 49, pp. 487-498, 2002.

[20] C. J. Valduga, D. C. Fernandes, A. C. Lo Prete, C. H. M. Azevedo, D. G. Rodrigues, and R. C. Maranhão, "Use of a cholesterol-rich microemulsion that binds to low-density lipoproteins receptors as vehicle for etoposide," Journal of Pharmacy and Pharmacology, vol. 55, no. 12, pp. 1615-1622, 2003.

[21] D. G. Rodrigues, D. A. Maria, D. C. Fernandes et al., "Improvement of paclitaxel therapeutic index by derivatization and association to a cholesterol-rich microemulsion: in vitro and in vivo studies," Cancer Chemotherapy and Pharmacology, vol. 55, no. 6, pp. 565-576, 2005.

[22] N. Nishiyama and K. Kataoka, "Current state, achievements, and future prospects of polymeric micelles as nanocarriers for drug and gene delivery," Pharmacology and Therapeutics, vol. 112, no. 3, pp. 630-648, 2006.

[23] L. Serpe, M. G. Catalano, R. Cavalli et al., "Cytotoxicity of anticancer drugs incorporated in solid lipid nanoparticles on HT-29 colorectal cancer cell line," European Journal of Pharmaceutics and Biopharmaceutics, vol. 58, no. 3, pp. 673680, 2004.

[24] X. G. Zhang, J. Miao, Y. Q. Dai, Y. Z. Du, H. Yuan, and F. Q. Hu, "Reversal activity of nanostructured lipid carriers loading cytotoxic drug in multi-drug resistant cancer cells," International Journal of Pharmaceutics, vol. 361, no. 1-2, pp. 239-244, 2008.

[25] M. K. Lee, S. J. Lim, and C. K. Kim, "Preparation, characterization and in vitro cytotoxicity of paclitaxel-loaded sterically stabilized solid lipid nanoparticles," Biomaterials, vol. 28, no. 12, pp. 2137-2146, 2007.

[26] R. Li, J. S. Eun, and M. K. Lee, "Pharmacokinetics and biodistribution of paclitaxel loaded in pegylated solid lipid nanoparticles after intravenous administration," Archives of Pharmacal Research, vol. 34, no. 2, pp. 331-337, 2011.

[27] H. Yuan, J. Miao, Y. Z. Du, J. You, F. Q. Hu, and S. Zeng, "Cellular uptake of solid lipid nanoparticles and cytotoxicity of encapsulated paclitaxel in A549 cancer cells," International Journal of Pharmaceutics, vol. 348, no. 1-2, pp. 137-145, 2008.

[28] D. Liu, Z. Liu, L. Wang, C. Zhang, and N. Zhang, "Nanostructured lipid carriers as novel carrier for parenteral delivery of docetaxel," Colloids and Surfaces B, vol. 85, no. 2, pp. 262-269, 2011.

[29] F. Q. Hu, Y. Zhang, Y. Z. Du, and H. Yuan, "Nimodipine loaded lipid nanospheres prepared by solvent diffusion method in a drug saturated aqueous system," International Journal of Pharmaceutics, vol. 348, no. 1-2, pp. 146-152, 2008.

[30] F. Q. Hu, S. P. Jiang, Y. Z. Du, H. Yuan, Y. Q. Ye, and S. Zeng, "Preparation and characterization of stearic acid nanostructured lipid carriers by solvent diffusion method in an aqueous system," Colloids and Surfaces B, vol. 45, no. 3-4, pp. 167-173, 2005.

[31] R. H. Müller, M. Radtke, and S. A. Wissing, "Solid lipid nanoparticles (SLN) and nanostructured lipid carriers (NLC) in cosmetic and dermatological preparations," Advanced Drug Delivery Reviews, vol. 54, pp. S131-S155, 2002.

[32] J. Varshosaz, S. Eskandari, and M. Tabbakhian, "Freeze-drying of nanostructure lipid carriers by different carbohydrate polymers used as cryoprotectants," Carbohydrate Polymer, vol. 88, pp. 1157-1163, 2012.

[33] C. Freitas and R. H. Müller, "Effect of light and temperature on zeta potential and physical stability in solid lipid nanoparticle
(SLN) dispersions," International Journal of Pharmaceutics, vol. 168, no. 2, pp. 221-229, 1998.

[34] S. J. Lim and C. K. Kim, "Formulation parameters determining the physicochemical characteristics of solid lipid nanoparticles loaded with all-trans retinoic acid," International Journal of Pharmaceutics, vol. 243, no. 1-2, pp. 135-146, 2002.

[35] V. C. F. Mosqueira, P. Legrand, J. L. Morgat et al., "Biodistribution of long-circulating PEG-grafted nanocapsules in mice: effects of PEG chain length and density," Pharmaceutical Research, vol. 18, no. 10, pp. 1411-1419, 2001.

[36] E. B. Souto, S. A. Wissing, C. M. Barbosa, and R. H. Müller, "Development of a controlled release formulation based on SLN and NLC for topical clotrimazole delivery," International Journal of Pharmaceutics, vol. 278, no. 1, pp. 71-77, 2004.

[37] A. Saez, M. Guzmán, J. Molpeceres, and M. R. Aberturas, "Freeze-drying of polycaprolactone and poly(D,L-lacticglycolic) nanoparticles induce minor particle size changes affecting the oral pharmacokinetics of loaded drugs," European Journal of Pharmaceutics and Biopharmaceutics, vol. 50, no. 3, pp. 379-387, 2000. 

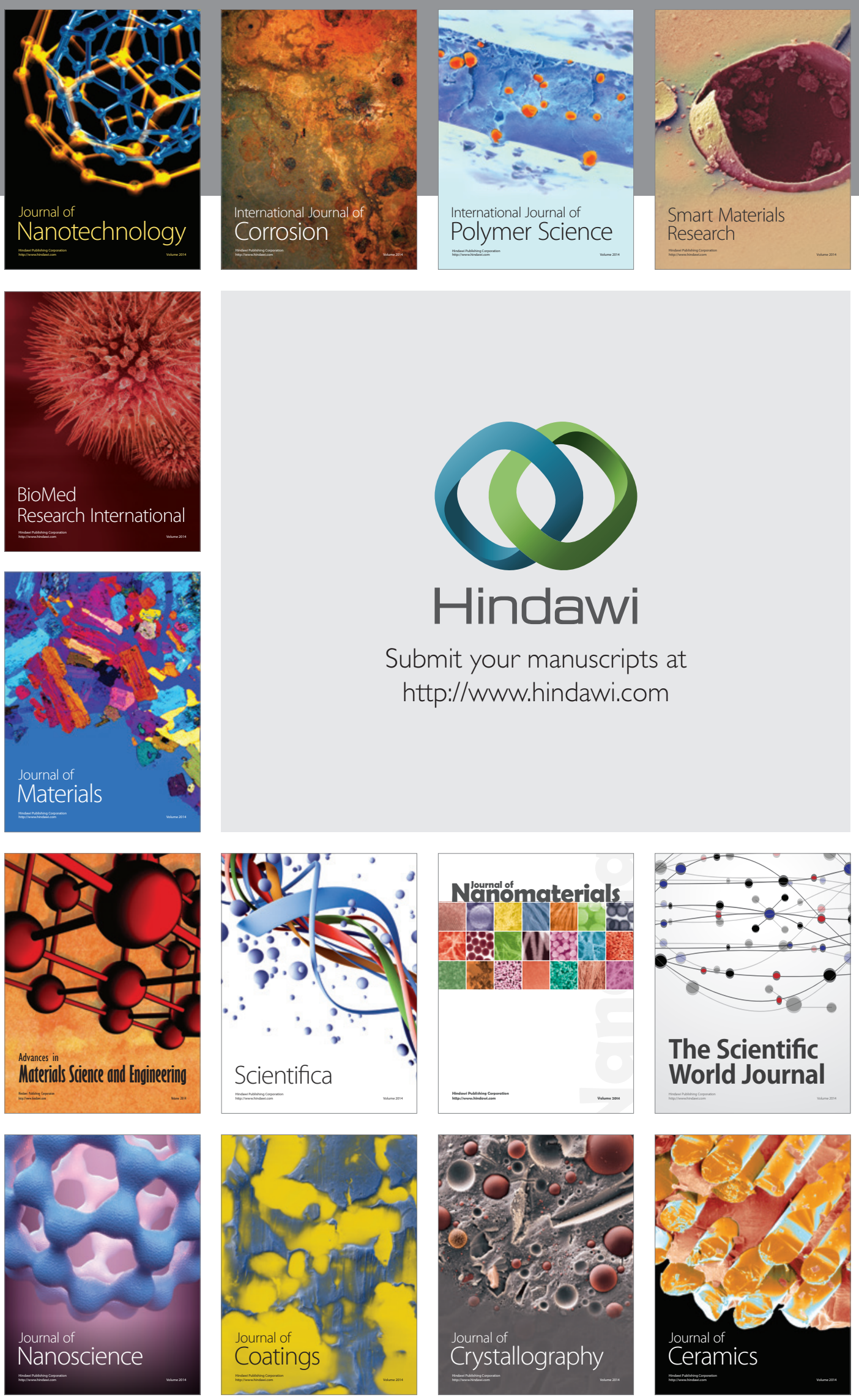

The Scientific World Journal

Submit your manuscripts at

http://www.hindawi.com

\section{World Journal}

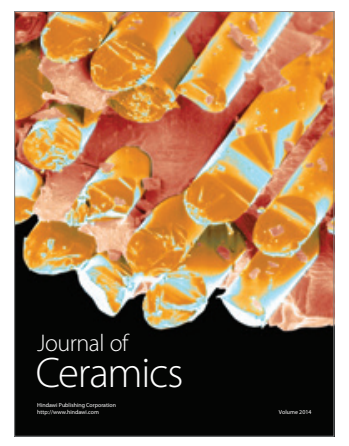

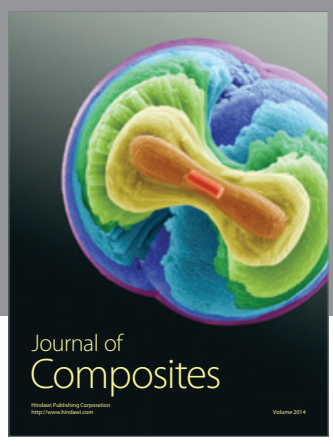
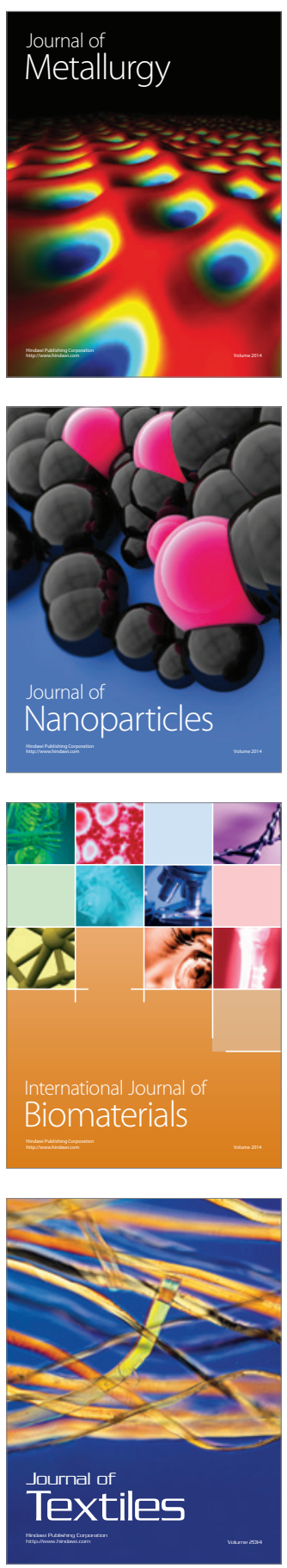